schizencephaly, and other heterotopias of the gray matter are being recognized more frequently by the use of the magnetic resonance imaging technique. The role of neurosurgical treatment in patients with neuronal migration disorders is being explored. The early recognition and effective surgical management of these cases might improve prognosis and prevent the development of intractable epilepsy and mental deterioration.

\title{
DEVELOPMENTAL DYSPHASIA SUBTYPES
}

The syndrames of developmental dysphasia and their remediation are outlined in the selected proceedings of the Fourth International Child Neurology Congress held in Jerusalem, Israel March 16-20, 1986. Six dysphasic syndromes are identified through the cambined psycholinguistic/ aphasiology model: 1) Verbal dyspraxia, 2) phonological production deficit, 3) verbal auditory agnosia, 4) phonologic-syntactic deficit, 5) lexical-syntactic deficit, and 6) semantic pragmatic deficit. In subtypes 1 and 2 impairment is in the expressive system with comprehension relatively intact. Verbal dyspraxia is a severe expressive disorder in which the child is extremely nonfluent and may be mute. Phonological production deficit is characterized by fluency but nonetheless unintelligibility. In both syndromes the production of consonants is more difficult than of vowels. Apraxia of speech and developmental misarticulation are terms sometimes applied to these expressive dysphasias. All dyspraxic dysphasias are difficult to treat and many require speech and language therapy beyond the preschool years. Many need to be introduced to a visual-manual system such as reading and writing at an early age, and some severe cases require formal sign language in conjunction with verbalization. Subtype 3 verbal auditory agnosia, camonly called word deafness, is an inability to decode spoken language at the level of phonology. Despite normal peripheral hearing the individual is unable to derive any meaning from the sounds he or she hears. These children comprehend virtually nothing and are essentially mute. Naming an object, drawing a picture of the object, and naming the picture with a printed label facilitates vocabulary building and the labels can then be used to make requests. Subtype 4 is a mixed receptive expressive deficit and results in telegraphic speech with amission of prepositions, verbs, and the endings on nouns and verbs. The use of gestures, cammication boards and written words to supplement oral language are encouraged. In dysphasia subtype 5, lexical syntactic deficit, syntax or the arrangement of words is immature and comprehension of abstract language may be poor. Language is dysfluent with multiple hesitations, false starts and self-corrections. The syndrome is seen in both autistic and nonautistic children. Dysphasia subtype 6, semantic pragmatic deficits with hyperverbal or loquacious speech but their commumicative intent and semantic content are limited and superficial. Jargon, neologisms, paraphasias, and circumlocutions are camon. Speech is quite rapid and "cluttered". The subtyping of dysphasic children permits the design of specific interventions for the particular language needs of the children in each group. (Allen DA, Mendelson L, Rapin, I. Syndrame specific remediation in preschool developmental dysphasia in Child Neurology and Developmental Disabilities, edited by French, JH, Harel, S, Casaer, P. Baltimore, London, Sydney, Toronto. Paul H. Brookes Publishing Campany, 1989). 
COMMENT. These attempts to subtype dysphasic children are helpful in the definition of pathogenesis and brain localization and they allow the development of specific intervention strategies. The neurologic bases of developmental dysphasias are probably multiple. Same syndromes may result from genetic abnormalities in brain development while early focal or multifocal acquired brain pathology may be responsible for others. The differentiation of developmental and acquired aphasias in young children may be difficult and subtle cerebral abnormalities may be uncovered by the MRI in children who have otherwise normal neurologic findings. The authors of this article hypothesize that in the course of providing sound therapeutic intervention at an early age they may be able to enhance brain reorganization through the development of altermative pathways. For example, providing dysphasic children of subtype verbal auditory agnosia with a visual language system may promote a bypass of areas of auditory dysfunction and enable the children to acquire verbal language that would have been inaccessible through the auditory channel alone. Failure to show improvement after intensive remediation may be explained by bilateral brain abnormalities that prohibit reorganization. Aut istic children who exhibit severe social attentional and behavioral deficits have the most guarded prognosis.

\section{PROGNOSIS OF EXPRESSIVE LANGUAGE DELAY}

The rate of improvement and it's predictive factors have been studied in 26 two year old children with expressive language disorder at the Departments of Pediatrics and Psychology, State University of New York at Stony Brook, and Department of Child Development and Family Relations, University of North Carolina-Greensboro. After a five month follow-up improvement in expressive language was variable, with approximately $1 / 3$ of the children showing no improvement, $1 / 3$ with mild improvement, and $1 / 3$ in the normal range. Three variables measured by the pretest evaluation could be used to predict improvement within the five month period: 1) The size of the child's vocabulary with a cutoff score of $8 ; 2$ ) whether the child was reported as eating regular meals; and 3 ) the extent that the child engaged in quiet "other" behaviors during the mother-child interaction. The more vocabulary the child had used at hame, the more improvement in expressive language was seen. If the child was characterized as not eating three regular meals a day, the less improvement in expressive language was seen. Children who engaged in more periods of quiet noninteractive activity during play and cleanup situations were more likely to improve in expressive language. The reported vocabulary size was $81 \%$ accurate in identifying children's improvement and the prediction of improvement in growth of language. (Fischel, JE et al. Language growth in children with expressive language delay. Pediatrics February 1989; 83:218-227).

COMENT. Expressive language delay is not self-correcting in a short term period of five months for many two year old children. A pediatrician or other professional who routinely advises parents of two year old children with expressive language delay to wait for spontaneous improvement would be wrong $39 \%$ of the time. However, 